\title{
AN IMPLEMENTATION OF SMOOTHED PARTICLE HYDRODYNAMIC METHODS FOR FLUIDS PROBLEMS
}

\author{
Nguyen Hoai Son, Nguyen Duy Hung \\ University of Technical Education Ho Chi Minh City
}

\begin{abstract}
In this article, we present a numerical Smoothed Particle Hydrodynamic (SPH) method. In the SPH method for the Navier - Stokes equations the most widespread method to solve for pressure and mass conservation is the weakly compressible assumption (WCSPH). This article presents two important benchmark problems to validate the algorithm of SPH method. The two benchmark problems chosen are the Lid driven cavity problem and Poiseuille flow problem at very low Reynolds numbers. The $\mathrm{SPH}$ results are also in good agreement with the analytical solution.
\end{abstract}

\section{INTRODUCTION}

Smoothed particle hydrodynamic method ( $\mathrm{SPH}$ ) is a fully Lagrangian method, which does not require the use of any mesh. It was originally invented to simulate astrodynamics (Lucy 1977, Gingold \& Monaghan 1977 ). Since then the use of SPH has expanded in many areas of solid and fluid dynamics (involving large deformations, impacts, free-surface and multiphase flows). A major advantage of SPH over Eulerian methods is that the method does not need a grid to calculate spatial derivatives. Instead, they are found by summation of analytical differentiated interpolation formulae (Monaghan, 1992). The momentum and energy equations become sets of ordinary differential equations which are easy to understand in mechanical and thermodynamic terms. For example, the pressure gradient becomes a force between pairs of particles [4].

While Eulerian methods have difficulties to construct a mesh for the simulation domain when it has very complex interfaces, $\mathrm{SPH}$ is able to do it without any special front tracking treatment. Moreover, the convection term of Navier-Stokes equations can cause many problems in the Eulerian framework, which are only partially circumvented by introducing numerical diffusion when it is discrete. However, in SPH this term is implicitly considered.

\section{SPH FORMULATIONS}

In $\mathrm{SPH}$, the fundamental principle is to approximate any function $f(r)$

$$
f(r)=\int f\left(r^{\prime}\right) W\left(\vec{r}-\vec{r}^{\prime}, h\right) d \vec{r}^{\prime}
$$


where $h$ is called the smoothing length and $W\left(\vec{r}-\vec{r}^{\prime}, h\right)$ is the weighting function or kernel. This approximation, in discrete notation, leads to the following approximation of the function at a particle (interpolation point) $\underline{a}$,

$$
f(r)=\sum_{b} m_{b} \frac{f_{a}}{\rho_{b}} W_{a b}
$$

where the summation is over all the particles within the region of compact support of the kernel function. The mass and density are denoted by $m_{b}$ and $\rho_{b}$ respectively and $W_{a b}=W\left(\vec{r}_{a}-\vec{r}_{b}, h\right)$ is the weight function or the kernel function.

\section{THE KERNEL FUCNTION}

By considering a domain of interest $\Omega$, the kernel function $W_{h}$ must satisfy the three following properties:

- The kernel summation over the whole domain must be equal to unity:

$$
\int_{\Omega} W\left(r-r^{\prime}\right) d r^{\prime}=1
$$

- When the smoothing length $h$ tends to zero, the kernel function must tend to Dirac distribution:

$$
\lim _{h \rightarrow 0} W\left(x-x^{\prime}, h\right)=\delta\left(x-x^{\prime}\right) .
$$

- The kernel must be at least once differentiable and its derivative must be continuous in order to avoid large fluctuations which would affect the solution [1].

$$
W\left(x-x^{\prime}, h\right)=0 \quad \text { when } \quad\left|x-x^{\prime}\right|>\kappa h
$$

$\kappa$ is a coefficient which depends on the order of kernel.

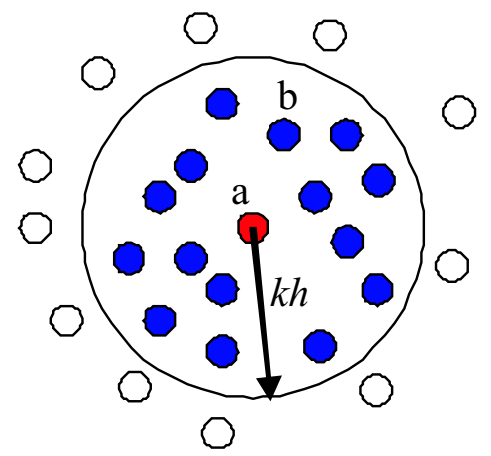

Fig. 1. Neighbor of particle with a compact support kernel.

The examples of $2^{\text {rd }}$ and $3^{\text {rd }}$ order of spline kernels function for 2-D are as follows:

$$
W(r, h)=\kappa \begin{cases}1-\frac{3}{2} q^{2}+\frac{3}{4} q^{3} & 0 \leq q \leq 1 \\ \frac{1}{4}(2-q)^{3} & 1 \leq q \leq 2 \\ 0 & q \geq 2\end{cases}
$$


where: $\kappa$ is $\frac{10}{7 \pi h^{2}}$ in $2 \mathrm{D}$ and $\frac{1}{\pi h^{3}}$ in $3 \mathrm{D} ; q=\frac{r}{h}, r$ being the distance between particles $a$ and $b$.

\section{THE CHOICE OF SMOOTHING LENGTH}

The smoothing length $h$ is very important in the SPH method, which has direct influence on the efficiency of the computation and the accuracy of the solution. If the smoothing length is too large, all details of the particle or local properties may be smoothed out, and the accuracy suffers, too. The particle approximations used by the SPH method depend on having a sufficient and necessary number of particles within the support domain of $k h$. There are many ways to dynamically evolve $h$ so that the number of the neighboring particles remains relatively constant. The simplest approach is to update the smoothing length according to the average density:

$$
h=h_{0}\left(\frac{\rho_{0}}{\rho}\right)^{1 / d}
$$

where $h_{0}$ and $\rho_{0}$ are the initial smoothing length and the initial density respectively, $d$ is the number of dimensions.

\section{CONTINUITY, MOMENTUM AND STATE EQUATION}

Density can be calculated directly from the SPH interpolation formulation (2):

$$
\rho_{a}=\sum_{b} m_{b} w_{b}\left(r_{a b}\right)
$$

By solving the continuity equation with one if the SPH divergence forms:

$$
\frac{d \rho_{a}}{d t}=\sum_{b} m_{b} \bar{u}_{a b} . \nabla_{a} w_{h}\left(r_{a b}\right)
$$

where $\bar{u}_{a b}=\bar{u}_{a}-\bar{u}_{b}$. According to Monaghan [16]. The equation (8) has advantages over (7). Density with (7) drops significantly near the edge of the fluid, which is unphysical. However, density obtained from (8) will vary only when particles move relative to each other. There is also a computational advantage in using (8) since the rates of change of all physical variables can be computed in one subroutine.

The momentum conservation equation in a continuum field is [5]:

$$
\frac{D \vec{v}}{D t}=-\frac{1}{\rho} \nabla P+\vec{g}+\vec{\Theta}
$$

where $\vec{\Theta}$ refers to the diffusion terms.

Different approaches, based on various existing formulations of the diffusive terms, can be considered in the SPH method to describe the momentum equation. Two different options for diffusion are focused: (i) artificial viscosity, (ii) laminar viscosity.

(i) artificial viscosity 
The artificial viscosity proposed by Monaghan (1992). In SPH notation, Eq. 10 can be written as follow:

$$
\frac{d \vec{v}_{a}}{d t}=-\sum_{b} m_{b}\left(\frac{\bar{p}_{b}}{\rho_{b}^{2}}+\frac{\bar{p}_{a}}{\rho_{a}^{2}}+\Pi_{a b}\right) \nabla_{a} W_{a b}+\vec{g}
$$

The pressure gradient term in symmetrical form is expressed in SPH notation as

$$
\left(-\frac{1}{\rho_{a}} \nabla P\right)=-\sum_{b} m_{b}\left(\frac{P_{b}}{\rho_{b}^{2}}+\frac{P_{a}}{\rho_{a}^{2}}\right) \nabla_{a} W_{a b}
$$

With $P_{k}$ and $\rho_{k}$ are the pressure and density corresponding to particle $k$ (evaluated at $\underline{\mathrm{a}}$ or $\underline{\mathrm{b}})$.

$$
\Pi_{a b}=\left\{\begin{array}{cc}
\frac{-\alpha \bar{c}_{a b} \mu_{a b}}{\bar{\rho}_{a b}} & \vec{v}_{a b} \vec{r}_{a b}<0 \\
0 & \vec{v}_{a b} \vec{r}_{a b}>0
\end{array}\right.
$$

where $\Pi_{a b}$ is the viscosity term with $\mu_{a b}=\frac{h \vec{\nu}_{a b} \vec{r}_{a b}}{\vec{r}_{a b}^{2}+\eta^{2}}$; where $\vec{r}_{a b}=\vec{r}_{a}-\vec{r}_{b}, \vec{\nu}_{a b}=\vec{\nu}_{a}-\vec{\nu}_{b}$; being $\vec{r}_{k}$ and $\vec{v}_{k}$ the position and velocity corresponding to particle $\mathrm{k}$ ( $\underline{\mathrm{a}}$ or $\underline{\mathrm{b}}$ ); $\bar{c}_{a b}=$ $\frac{c_{a}+c_{b}}{2}, \eta^{2}=0.01 \mathrm{~h}^{2}, \alpha$ is a free parameter that can be changed to each problem.

(ii) laminar viscosity.

The momentum conservation equation with laminar viscous stresses is given by

$$
\frac{D \vec{v}}{D t}=-\frac{1}{\rho} \nabla P+\vec{g}+v_{o} \nabla^{2} \vec{v}
$$

where the laminar stress term simplifies (Morris et at., 1997) to:

$$
\left(v_{o} \nabla^{2} \bar{v}\right)_{a}=\sum_{b} m_{b}\left(\frac{4 v_{o} \vec{r}_{a b} \nabla_{a} W_{a b}}{\left(\rho_{a}+\rho_{b}\right)\left|\vec{r}_{a b}^{2}\right|}\right) \vec{v}_{a b}
$$

where $v_{o}$ is the kinetic viscosity of laminar flow $\left(10^{-6} \mathrm{~m} / \mathrm{s}^{2}\right)$.

Following (Monaghan et al., 1999; Batchelor, 1974), the relationship between pressure and density is assumed to follow the expression:

$$
P=B\left[\left(\frac{\rho}{\rho_{0}}\right)^{\gamma}-1\right]
$$

where $\gamma=7$ and $B=c_{0}^{2} \rho_{0} / \gamma$ being $\rho_{0}=1000 \mathrm{~kg} / \mathrm{m}^{3}$ the reference density and $c_{0} c\left(\rho_{0}\right)=$ $\left.\sqrt{\left(\frac{\partial P}{\partial \rho}\right)}\right|_{\rho_{0}}$ the speed of sound at the reference density.

\section{BOUNDARY CONDITIONS}

The boundary conditions are implemented using either dynamic boundary conditions (Crespo et al, 2007) and repulse boundary conditions (Monaghan \& Kos, 1999). Dynamic boundary particle are forced to satisfy the same equations as fluid particles. Thus, they follow the momentum equation, the continuity equation, the equation of state and energy equation. They remain fixed in position (fixed boundary). Repulse boundary 
condition was developed by Monaghan (1994) to ensure that a water particle can never cross a solid boundary.

\section{NUMERICAL EXAMPLE}

\section{Lid - driven cavity problem}

The first benchmark problem solved using SPH method is a two - dimensional simulation of a lid - driven cavity problem. The lid - driven cavity problem is the fluid flow within a closed square generated by moving the top side of the square at a constant velocity $V_{\text {top }}$ while the other three side stationary. The flow will reach steady state and form a recirculation pattern. In the simulation, the dimension of the side of the square domain is $l=10^{-3} \mathrm{~m}$, kinetic viscosity and density are $v=10^{-6} \mathrm{~m}^{2} / \mathrm{s}$ and $\rho=1000$ $\mathrm{kg} / \mathrm{m}^{3}$ respectively. The top side of the square moves at a velocity of $U_{\mathrm{Lid}}=10^{-3} \mathrm{~m} / \mathrm{s}$, thus the Reynolds number for this case is 1 .

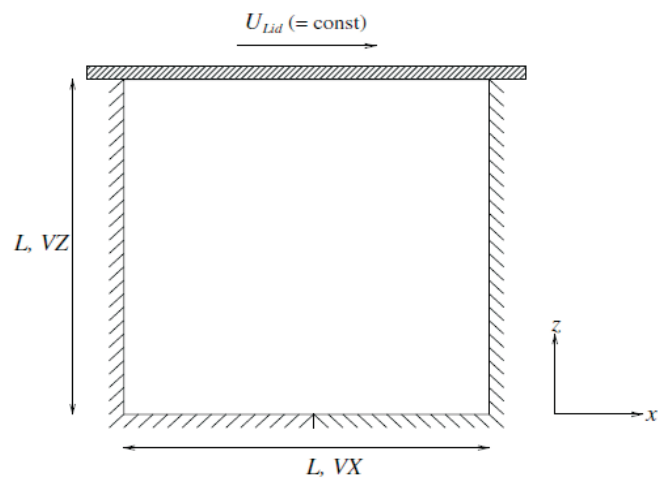

Fig. 2. Two - dimension lid - driven cavity flow domain

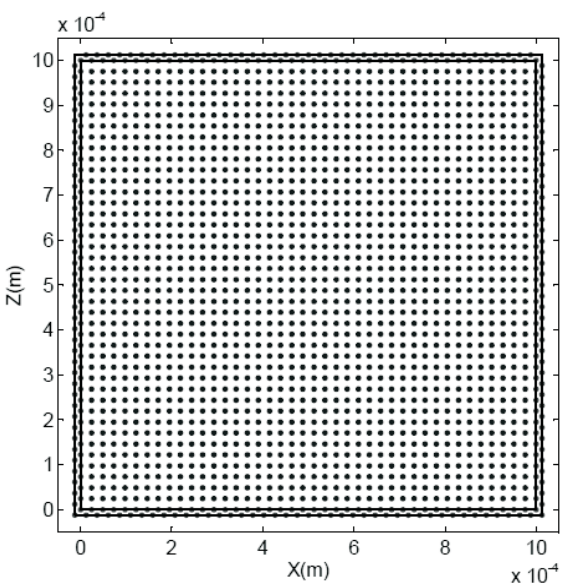

Fig. 3. Initial particle distributions. The interior fluid particle (dot) and boundary particle (dot line). With array $40 \times 40$ particles.

Figure 3 show the velocity distridution at the steady state, in which the recirculation parttern of flow can be observed clearly. Figure 4 shows the non - dimentional vertical velocity profile along the horizontal centerline, while Figure 5 shows the non - dimensional horizontal velocity profile along the vertical centerline. It can be seen from Table 1 that results from the present method and those from the finite differences method by Marques \& Doricio [16] or R. Schreiber \& Keller [15] agree well.

Table 1. Compare location of primary vortex center.

\begin{tabular}{|l|l|l|l|l|l|}
\hline Schreiber \& Keller [15] & \multicolumn{4}{l|}{ Marques \& Doricio [16] } & \multicolumn{2}{l|}{ Present } \\
\hline $\mathrm{x}$ & $\mathrm{z}$ & $\mathrm{x}$ & $\mathrm{z}$ & $\mathrm{x}$ & $\mathrm{z}$ \\
\hline 0.50 & 0.76667 & 0.4906 & 0.7624 & 0.491 & 0.765 \\
\hline
\end{tabular}




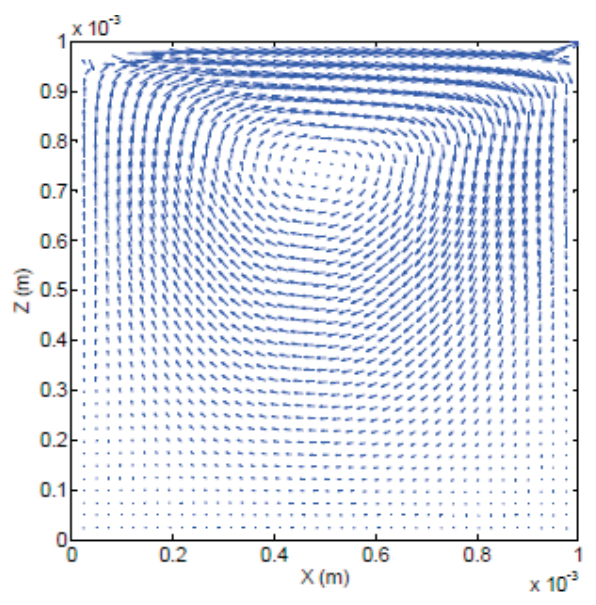

Fig. 4. Steady state velocity distributions for lid - driven cavity flow. The length of the arrows represents the magnitude of the velocity for $\operatorname{Re}=1$ (scale $=2$ )

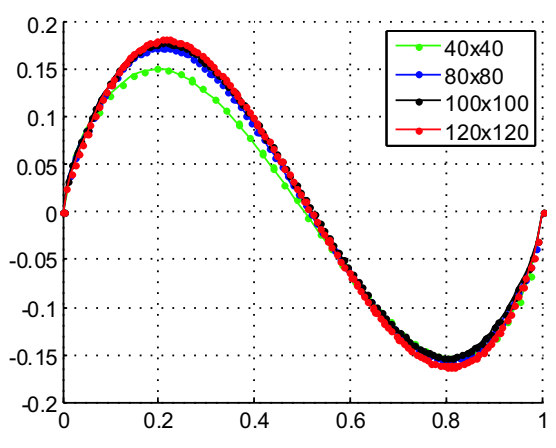

Fig. 5. Non - dimension vertical velocities along the horizontal centerline

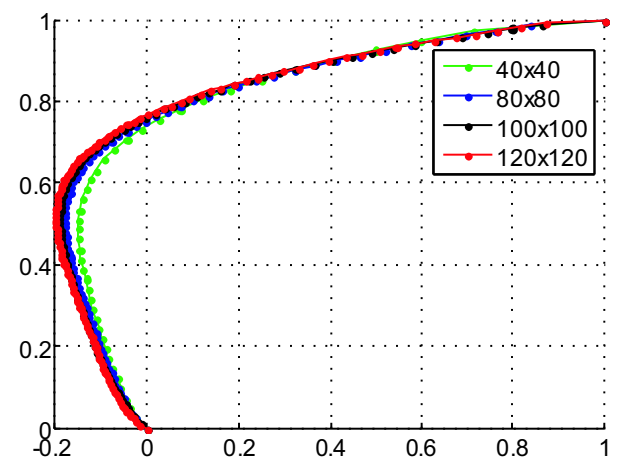

Fig. 6. Non - dimension horizontal velocities along the vertical centerline

\section{Poiseuille flow problem}

The Poiseuille problem consists of a fluid between flow fixed plate placed at $z=0$ and $z=10^{-3} \mathrm{~m}$. Initial the fluid is at rest while for $t>0$ a volume force $F$ is acting parallel to $x$ - direction, In this problem, $l=10^{-3} \mathrm{~m}, \rho=1000 \mathrm{~kg} / \mathrm{m}^{3}$, the kinetic viscosity $v=10^{-6} \mathrm{~m}^{2} / \mathrm{s}$ and the driven body force $F=2 \times 10^{-4} \mathrm{~m} / \mathrm{s}^{2}$.

We have [1]: $\quad v x(z)=-\frac{F}{2 v}\left(a^{2}-z^{2}\right)$, where $a=l / 2, z= \pm l / 2$

According to equation (1), the peak fluid velocity is $v_{0}=2.5 \times 10^{-5} \mathrm{~m} / \mathrm{s}$, which correspond to a

Reynolds number of $\operatorname{Re}=2.5 \times 10^{-2}$ according to the following expression:

$$
\operatorname{Re}=\frac{v_{0} l}{v}
$$



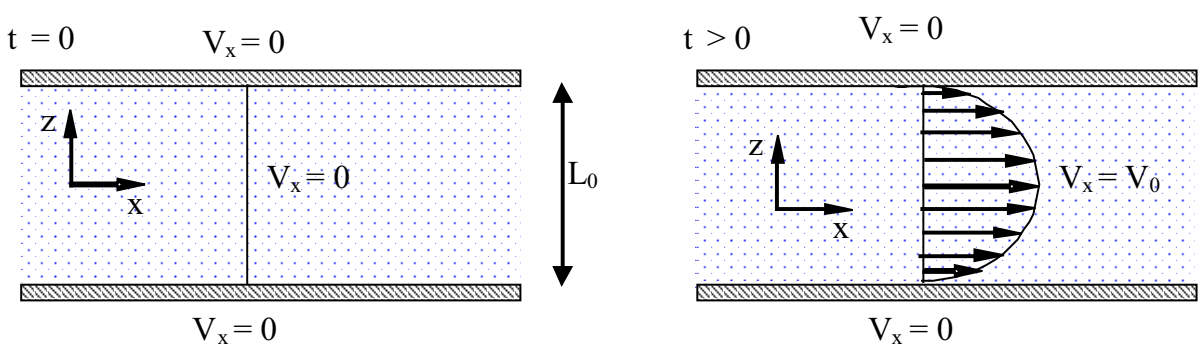

Fig. 7. Poiseuille flow domain centerline

The Navier - Stokes equations for this problem, written in dimensionless form:

$$
\frac{d \vec{v}_{a}}{d t}=-\sum_{b} m_{b}\left(\frac{\bar{p}_{b}}{\rho_{b}^{2}}+\frac{\bar{p}_{a}}{\rho_{a}^{2}}+\Pi_{a b}\right) \nabla_{a} W_{a b}+\sum_{b} m_{b}\left(\frac{4 v_{o} \vec{r}_{a b} \nabla_{a} W_{a b}}{\left(\rho_{a}+\rho_{b}\right)\left|\vec{r}_{a b}^{2}\right|}\right) \vec{v}_{a b}+F_{x}
$$

Morris et at. (1997) provided a series solution for the time dependent behavior of the Poiseuille flow [1]

$$
v_{x}(z, t)=\frac{F}{2 v} z(z-l)+\sum_{n=0}^{\infty} \frac{4 F l^{2}}{v \pi^{3}(2 n+1)^{3}} \sin \left(\frac{\pi z}{l}(2 n+1)\right) \exp \left(-\frac{(2 n+1)^{2} \pi^{2} v}{l^{2}} t\right)
$$

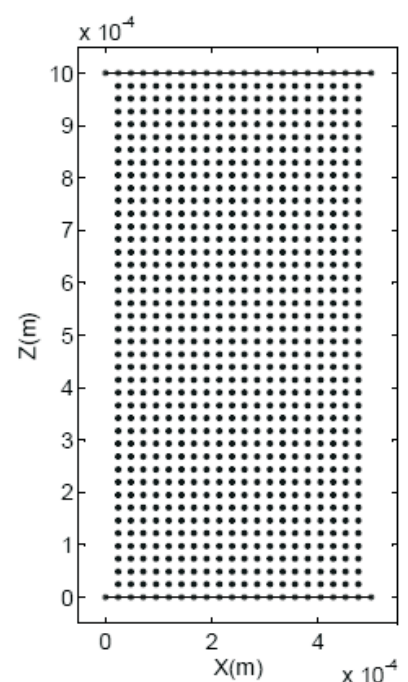

Fig. 8. Initial geometry and distribution for the Poiseuille flow with array $20 \times 40$ particles, (dot line: boundary particles, dot: fluid particles)

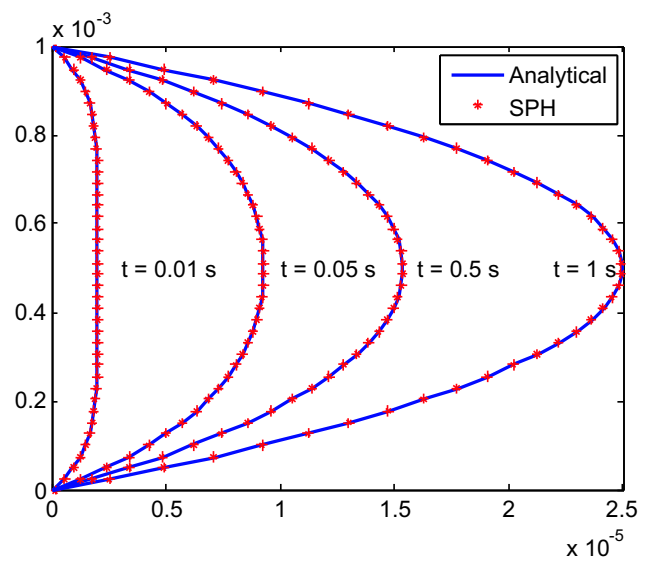

Fig. 9. Velocity profiles for Poiseuille flow

In Figure 9, we show the results of the transient behavior for a sequence of times until $1.0 \mathrm{~s}$, when the velocity profile reaches its results - state regime given by Eq. (18). The numerical solution (filled dots) is compared with the exact one (solid curves) as calculated 

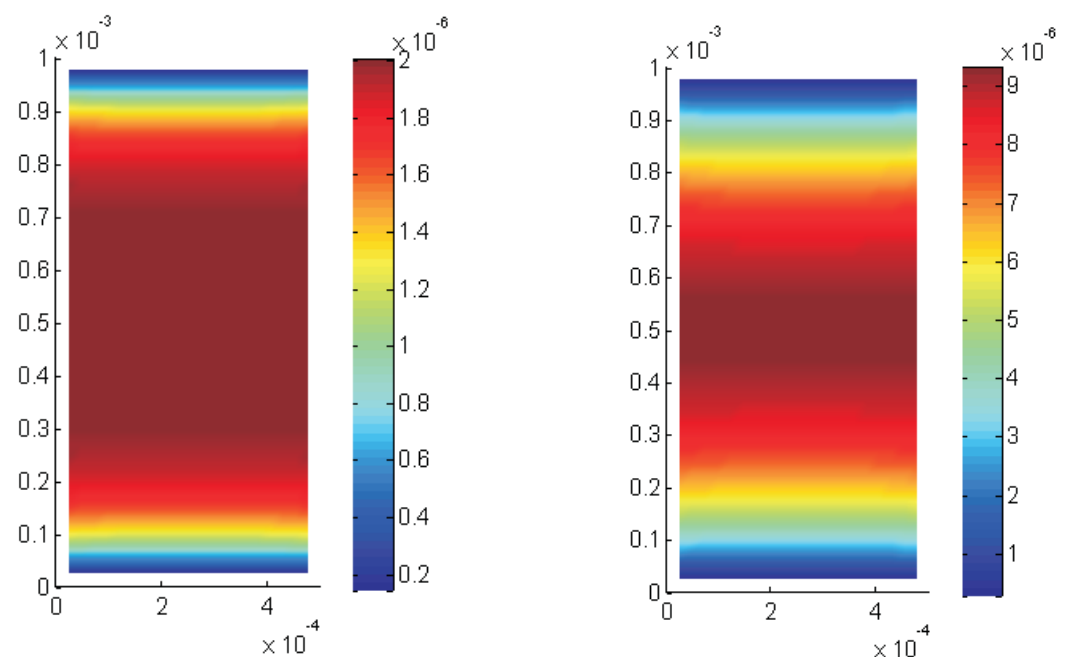
$\mathrm{t}=0.01 \mathrm{~s}, V_{\mathrm{xmax}}=2.0 \mathrm{e}-6 \mathrm{~m} / \mathrm{s}$, $V_{\mathrm{xmin}}=1.45 \mathrm{e}-7 \mathrm{~m} / \mathrm{s}$

$$
\begin{gathered}
\mathrm{t}=0.05 \mathrm{~s}, V_{\text {xmax }}=9.29 \mathrm{e}-6 \mathrm{~m} / \mathrm{s} \\
V_{\text {xmin }}=2.84 \mathrm{e}-7 \mathrm{~m} / \mathrm{s}
\end{gathered}
$$
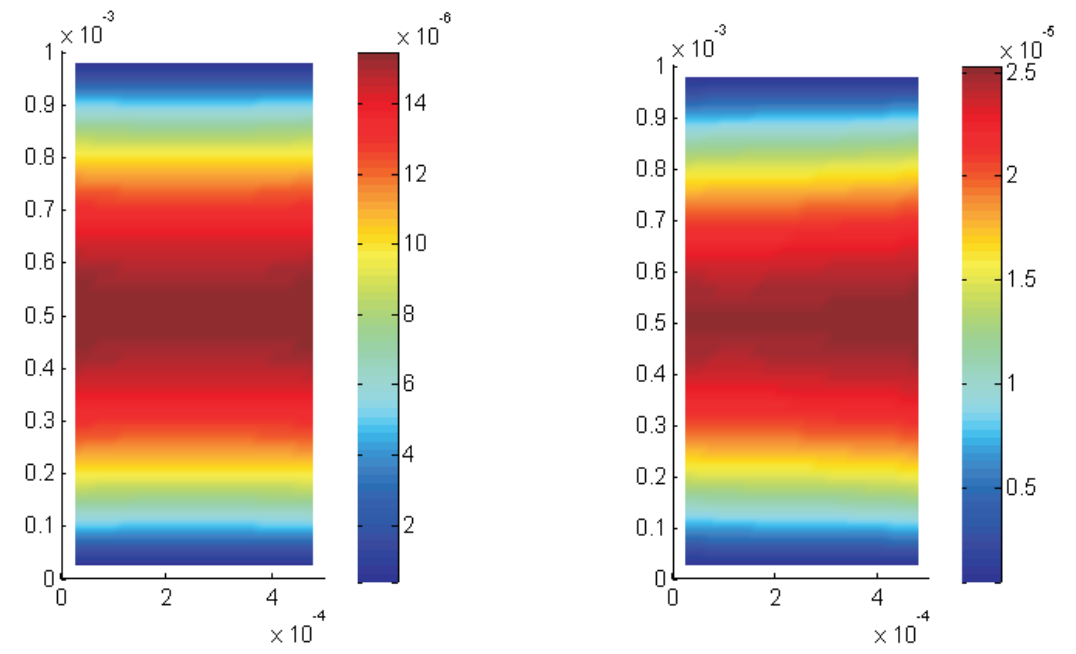

$$
\begin{aligned}
t= & 0.1 \mathrm{~s}, V_{\mathrm{x} \max }=1.54 \mathrm{e}-5 \mathrm{~m} / \mathrm{s} \\
& V_{\mathrm{x} \min }=3.78 \mathrm{e}-7 \mathrm{~m} / \mathrm{s}
\end{aligned}
$$

$$
\begin{gathered}
t=1 \mathrm{~s} V_{\text {xmax }}=2.49 \mathrm{e}-5 \mathrm{~m} / \mathrm{s} \\
V_{\text {xmin }}=4.78 \mathrm{e}-7 \mathrm{~m} / \mathrm{s}
\end{gathered}
$$

Fig. 10. Depicts the flow velocity distribution at time t $=0.05 \mathrm{~s}, 0.1 \mathrm{~s}, 0.5 \mathrm{~s}$ and $1.0 \mathrm{~s}$

from Eq. (19). The dots on each curve are obtained by averaging over neighboring particles. Compared with the exact solution, the maximum relative error in the velocity is less than $0.5 \%$ for the steady-state solution at $1.0 \mathrm{~s}$..

Furthermore, the maximum and minimum calculated densities are $\rho_{\max }=1.004 \rho_{0}$ and $\rho_{\min }=0.999 \rho_{0}$, respectively, so that the incompressibility of the flow is very well reproduced by the numerical scheme. 


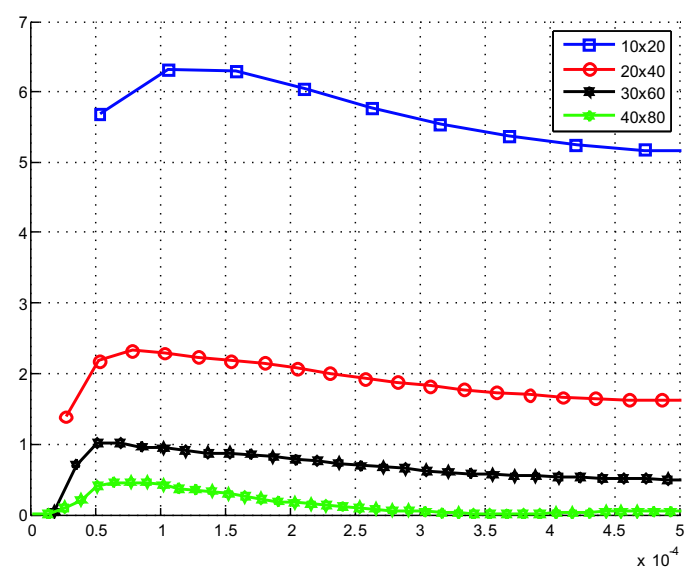

Fig. 11. Compare error of velocity with analytical solution using smoothing length as 1.05 times the initial particle spacing at $t=1.0 \mathrm{~s}$

\section{CONCLUSION}

- The errors estimate between SPH solution and exact solution are always underestimated $(0.5 \%)$ and the reliability is ensured for problems with singular point.

- The sub-domain algorithm and the implemented global/particle data structures enabled to use higher order particles ranging from order $(40,40),(80,80),(100,100)$ and $(120,120)$ are ensured the convergence of SPH method.

- Two important benchmark problems to validate that the SPH method produces results that agree with know numerical and experimental literature were in very good agreement.

- A little remark has to be mentioned about the numerical integration. To have accurate result, the numerical integration has to be very fine, i.e. many integration points. Note that it converge even with a small amount of integration points, but it is not accurate enough. The only requirement to converge is that the number of particles in the support domain is greater than the number of components in $p$. This to guarantee that the matrix $A$ is invertible. The many integration points combined with the enlarged system because of the Lagrange multiplier, results in a very time consuming program. Even for a so simple problem with linear static. But no optimization where made on the code to improve the performance.

\section{REFERENCES}

[1] G R Liu, M B Liu, Smoothed Particle Hydrodynamic - a meshfree particle method, World Scientific, 2003.

[2] M. Yildiz, R. A. Rook and A. Suleman, SPH with the multiple boundary tangent method, International journal for numerical methods in engineering, 2008.

[3] Leonardo Di G. Sigalotti, Jaime Klapp, Eloy Sira, SPH simulation of time - dependent Poiseuille flow at flow Reynolds numbers, Journal of Computational Physics 191 ( 2003) $622-628$ 
[4] E. S. Lee, C. Moulinec, R. Xu, D. Violeau, D. Laurence, P. Stansby, Comparisions of weakly compressible and truly incompressible algrorithm for SPH meshfree particle method, Journal of Computational Physics 227 ( 2008) 8417-8436.

[5] Alejandro Jacobo Cabrera Crespo, Application of the Smoothed Particle Hydrodynamics model SPHysics to free - surface hydrodynamics, PhD thesis, 2008.

[6] Joseph Peter Morris, Analysis of Smoothed Particle Hydrodynamics with Applications, Doctor of Phylosophy, 1996.

[7] Y. Vidal, Meshfree Methods for Dynamic Problems - Incompressibility and Large Strains, Doctural Thesis, 2004.

[8] V. Pertersson, An Implementation of Meshfree Methods for Mechanical Problems at Large Strains, Master Thesis, 2007.

[9] W. K. Liu, S. Li and T. Belytschko, Moving Least Quare Reproducing Kernel Methods, Computer Methods in Applied Mechanics and Engineering, 1996.

[10] G. R. Liu, Meshfree Methods: Moving beyond the Finite Element Method, CRC Press, 2003

[11] G. R. Liu and Y. T. Gu, An Introduction to Meshfree Methods and their Programming, Springer, 2005.

[12] J. Donea and A. Huerta, Finite Element Methods for Flow Problems, John Wiley \& Sons, 2003.

[13] P. K. Kundu and I. M. Cohen, Fluid Mechancis $2^{N D}$, Elservier, 2002.

[14] J.J. Monaghan, Smoothed Particle Hydrodynamics, Annu. Rev. Astron. Astrophy. 30 (1992) $543-574$.

[15] R. Schreiber \& Keller, Driven Cavity Flows by Efficient Numerical Techniques, Journal of Computational Physics 49 (1983) 310-333.

[16] H. Marques and L. Doricio, Numerical investigation of the flow in a two-dimensional cavity: meshless, finite volumes and finite differences methods, Latin American Journal of Solids and Structures 3 (2006) 301 - 324

[17] Website: http://wiki.manchester.ac.uk/spheric/index.php/SPHERIC_Home_Page

Received June 21, 2009

\section{ƯNG DUUNG PHƯƠNG PHÁP SMOOTHED PARTICLE HYDRODYNAMIC ĐỂ GIẢI CÁC BÀI TOÁN CƠ HỌC CHẤT LỎNG}

Một trong những phương pháp số giải quyết bài toán cơ học lưu chất được quan tâm trong bài báo này là phương pháp Smoothed Particle Hydrodynamic (SPH). Xây dựng nghiệm với giải thiết nén yếu của phương trình Navier - Stokes cho bài toán cơ học lưu chất dựa trên sự bảo toàn áp suất và khối lượng. Ở đây, phương pháp SPH được áp dụng để giải hai bài toán của cơ học chất lỏng: bài toán Lid - driven cavity và bài toán Poiseuille với hệ số Reynolds thấp. Kết quả từ phương pháp SPH được đánh giá và so sánh từ các lời giải chính xác hoặc dựa trên sai số chuẩn năng lượng với mật độ làm mịn khác nhau. 